\title{
COMMENTS ON THE REPORT OF THE COMMITTEE ON THE PROVISIONAL PROGRAMME FOR THE CONGRESS.
}

BY DR. JOHN SAHULKA, AUSTRIAN DELEGATE TO THE CONGRESS.

\section{[TRANSLATION.]}

I. New Units.

1. In the calculation of magnetic circuits the field strengths which occur in practice would have to be expressed in very small decimals, and magnetic resistances in very large numbers. In order to have convenient numbers, it would therefore be necessary to use the units micro-gauss and mega-oersted. This makes it desirable to retain the absolute c. G. s. system of units. The practical units volt, ampere and ohm, were introduced only because the absolute c. G. S. units would have given inconvenient figures for the quantities occuring in practice. In magnetic circuits no reason exists for giving up the absolute system of units.

2. 'The introduction of a unit (the mho) for the electrical conductivity of a circuit is not a necessity, as all calculations can be made with the units ohm, ampere and volt.

\section{Names for New UntTs.}

1. Should the absolute o. G. s. system of units for magnetic circuits be retained, which from recently expressed opinions seems probable, the introduction of new names would not be necessary.

2 . The name "mho" for the unit of electrical conductivity was probably used by some one because it was introduced by Sir Wm. Thomson; should the new unit be introduced, it would be easy to find an appropriate name (thomson). In order to be consistent, one would then have to introduce a practical unit for magnetic conductivity (reluctivity ${ }^{1}$ ), and give it a name which is the reverse of the name oersted.

3. The name henry for the practical unit of self and mutual induction is preferred to the term quadrant, because induction coefficients are not lengths.

1 Inductivity, or magnetic permeability, is doubtless what was meant. Tr. 


\section{DEFINITIONS.}

1. "The impressed electromotive force is the ratio of the total activity of an electrically conducting circuit to its instantaneous current strength." Formerly the term "impressed electromotive force" was understood to mean the difference of potential measured at an alternating current apparatus, or in case the whole circuit is considered, the total electromotive force. This cannot be meant by the proposed definition as the instantaneous current strength occurs in it. But if by impressed electromotive force is meant the instantaneous value of the difference of potential at the terminals or the electromotive force, there is a contradiction, for if, for instance, a circuit traversed by an alternating current contains a self-induction, then at the instant when the instan. taneous value of the electromotive force is equal to zero, neither the current nor the accumulated energy in the form of a magnetic field, are zero, and therefore the quotient of these values will likewise not be equal to zero; there is also a contradiction if a condenser is inserted in the circuit.

2. The universally used term permeability should not be replaced by the term inductivity.

3. It is very desirable that the definitions of induction resistance (inductance), and of the coefficient of self and mutual induction, be adopted in accordance with the suggestions of the Committee, taking into consideration the total field which is linked with a definite current strength, and not by taking into consideration the electromotive force induced during separate intervals of time. In the latter case the self-induction coefficient is a different one at every step of the magnetization, and is dependent on whether the magnetization increases or decreases. The change of value of the self-induction coefficient cannot be represented by a simple formula on account of the irregular form of the magnetization and hysteresis curve. Only such a definition of the self-induction coefficient is snitable in practice, according to which one can measure these values with the usual instruments and according to which one can make calculations. Therefore, of the three different ways in which the self-induction coefficient can be defined, only that one is adapted to practice which takes into consideration the total field which is generated. In order not to obtain incorrect values from the proposed definition, it should be added that the same is correct only for conductors which are not branched. Mr. Blondel has called attention to this feature. When a periodical alternating electromotive force, and not a constant one, is acting in a circuit, special relations arise which make it desirable to introduce also the quantities "effective ohmic resistance" (effective resistance), and "effective induced resistance" (effective inductance); only in the case of a coil withont iron, which exerts no action on any other conductor, are these quantities unnecessary. If a coil without iron is traversed by an alternating current, the difference of potential which acts 
can always be divided into two components, one of which overcomes the ohmic resistance, and the other the inductive resistance. The latter component precedes the former in phase by $90^{\circ}$; the overcoming of the inductive resistance requires the production of no energy. But if the coil contains an iron core, the energy of magnetization will have to be generated besides the heating effect of the current. In this case also there are two components of the difference of potential ; the one $E_{1}$ overcomes the ohmic resistance, the other $E_{2}$ overcomes the electromotive force induced by the periodically changing field in the coil. This component $E_{2}$ is no longer in advance of $E_{1}$ by a difference in phase of $90^{\circ}$, but by a smaller angle $\alpha$. The inductive resistance could be determined by dividing this second component $E_{2}$ by the current strength $I$; from this inductive resistance one would obtain the self-induction coefficient by dividing by $2 \pi n$. The component $E_{2}$ can also be resolved again into two components, of which the one $E_{2} \cos \alpha$ has the same phase as $E_{1}$, while the other $E_{2} \sin \alpha$ is advanced in phase by $90^{\circ}$. The component $E_{2} \cos \alpha$ involves the energy of the Foucault currents and hysteresis, the component $E_{2} \sin \alpha$ involves the expenditure of no energy. The coil with an iron core acts like a coil without iron which has the ohmic resistance $\left(E_{1}+E_{2}^{2} \cos \alpha\right) \div I$, and the indnctive resistance $\left(E_{2} \sin \alpha\right) \div I$. These values are the effective ohmic resistance (effective resistance), and the effective inductive resistance (effective inductance) of a coil with an iron core. In the same way one also obtains this effective resistance for every transformer, motor, condenser, etc. For the primary circuit of a transformer these values were calculated by Maxwell in the year 1865, and were represented in the above manner. The effective resistances of an alternating current apparatus can in every case be found when the current strength, the required difference of potential, and the energy consumed, are measured. The definitions of these values are as follows:

The effective resistance of a circuit is the ratio of the absorbed power to the mean square of the current.

The effective inductance is the square root of the difference of the squares of the impedance and the effective resistance.

The effective coefficient of self-induction is the ratio of the effective inductance to $2 \pi n$.

4. The definition of reluctivity cannot be given by saying:- - It is the reluctance per unit volume, as one does not obtain the total magnetic resistance by multiplying reluctivity by the volume; it would be preferable to have it read:- Reluctivity is the magnetic resistance of a portion of the material in question, having a length of $1 \mathrm{~cm}$. and a cross section of $1 \mathrm{~cm}$.

5. A definition of Mattheissen's standard is superfluous as the Congress is not concerned with researches regarding this unit.

Vranka, June 14th, 1893. 